Published in final edited form as:

Health Psychol. 2015 July ; 34(7): 779-782. doi:10.1037/hea0000174.

\title{
Life Satisfaction and Use of Preventive Health Care Services
}

\author{
Eric S. Kim, MS, \\ Department of Psychology, University of Michigan, Ann Arbor \\ Laura D. Kubzansky, PhD, and \\ Department of Social and Behavioral Sciences, Harvard School of Public Health, Boston, \\ Massachusetts \\ Jacqui Smith, PhD \\ Department of Psychology, University of Michigan, Ann Arbor; Institute for Social Research, \\ University of Michigan, Ann Arbor
}

\section{Abstract}

Objective-Although a growing body of research shows that life satisfaction is linked with enhanced health behaviors and physical health, no study to our knowledge has examined life satisfaction's association with use of preventive health care services. Building upon prior research we hypothesized that people with higher life satisfaction would be more proactive in taking care of their health, hence more likely to use preventive health care services.

Method-Multiple logistic regression models were used to examine the association between life satisfaction and preventive services. Participants were drawn from the Health and Retirement Study, a prospective and nationally representative panel study of adults over the age of 50. Their use of preventive services were collected over two years of follow-up.

Results-In models adjusting for sociodemographic factors, each standard deviation increase in life satisfaction was associated with a higher likelihood that people would obtain a cholesterol test $(\mathrm{OR}=1.07,95 \% \mathrm{CI}=1.00-1.16)$. Further, women with higher life satisfaction were more likely to obtain a mammogram/x-ray $(\mathrm{OR}=1.11,95 \% \mathrm{CI}=1.03-1.23)$, or pap smear $(\mathrm{OR}=1.14,95 \%$ $\mathrm{CI}=1.07-1.22)$, and also regularly check their breasts for lumps $(\mathrm{OR}=1.07,95 \% \mathrm{CI}=1.00-1.15)$, while men were more likely to obtain a prostate exam $(\mathrm{OR}=1.07,95 \% \mathrm{CI}=1.00-1.15)$.

Conclusion-Higher life satisfaction was associated with higher use of several preventive services. A growing body of randomized controlled trials that target life satisfaction have shown that levels of life satisfaction are modifiable. Thus, if these findings are replicated, life satisfaction may provide an important target for interventions aimed at enhancing preventive behaviors and health.

\section{Keywords}

life satisfaction; successful aging; health care utilization; preventive health care service; health screening; subjective well-being

Correspondence and reprint requests to: Eric S. Kim, Department of Psychology, 530 Church Street, University of Michigan, Ann Arbor, MI 48109-1043, USA; Phone: 734-647-3872; kimeric@umich.edu. 
A growing body of research shows that life satisfaction is prospectively associated with better health. Even after controlling for an array of risk factors, people with higher life satisfaction display healthier behaviors and other health advantages, such as enhanced cardiovascular health and longer lives (Boehm, Peterson, Kivimaki, \& Kubzansky, 2011; Diener \& Chan, 2011; Li, Ford, Zhao, \& Mokdad, 2009; Strine, Chapman, Balluz, Moriarty, \& Mokdad, 2008; Xu \& Roberts, 2010). Building on this evidence, recent research suggests that those with higher levels of life satisfaction are more proactive in taking care of their health; empirical evidence supports this hypothesis, demonstrating that life satisfaction is associated with fewer doctor visits over time (Kim, Park, Sun, Smith, \& Peterson, 2014). This research assumes that more doctor's visits are a proxy for poorer health, but in fact a more nuanced view may be appropriate. Since people visit the doctor either to address a problem, or in the service of prevention, simple measure assessing only the number of doctor visits a person makes may not distinguish between preventive or curative care. To test the hypothesis that life satisfaction may motivate greater willingness to engage in preventive health care and behaviors, we examined whether people with higher life satisfaction have a higher likelihood of using preventive health care services.

Less than $30 \%$ of adults aged 50-64 and less than 50\% of adults over the age of 65 are up-todate with recommended core preventive services (Centers for Disease Control, 2011; Department of Health and Human Services, 2010). Due to our rapidly growing population of older adults, who are more vulnerable to the threats of declining health and rising health care costs, identifying modifiable factors linked with increased use of preventive health care services in this age group is particularly important. Such an effort may be especially timely in the U.S. given the increased access to preventive care that has become available with the Affordable Care Act. Life satisfaction is shaped by social influences and changes throughout the life course (Diener, Lucas, \& Scollon, 2006; Lucas \& Donnellan, 2007). Further, two recent meta-analyses of randomized controlled trials, showed that various aspects of wellbeing (including life satisfaction) can be reliably and meaningfully enhanced, at least in young and midlife adults (Bolier et al., 2013; Sin \& Lyubomirsky, 2009). The more recent meta-analysis identified twelve randomized controlled trials that targeted life satisfaction. Out of the twelve studies, nine successfully modified satisfaction levels (Bolier et al., 2013). The diverse array of life satisfaction enhancing interventions included individual and group therapy, various online exercises, thinking about positive life events, expressing gratitude, and writing about positive events. The meta analysis by Bolier et al. (2013) also showed that the effects of these interventions lasted over a three to six month follow-up period (except in one study, participants were not tracked for longer than six months). While some interventions are more time-intensive, others are relatively brief and straightforward. Hence, they may be incorporated into practice more easily. Thus, with further research, life satisfaction may provide an important target for intervention aimed at enhancing health behaviors and health. 


\section{Methods}

\section{Study Design and Sample}

The Health and Retirement Study (HRS) is an ongoing nationally representative panel study of US adults over the age of 50. Since 1992, the HRS has interviewed over 37,000 Americans in biennial surveys. Starting in 2006, a random 50\% of active HRS respondents were initially assigned to a face-to-face interview that included the collection of biomarkers $(N=10,746)$. This assignment was made based on vital status in 2004 and prior to data collection in 2006. Respondents who completed the in-person interview were given a psychosocial questionnaire to complete in their own time and asked to return it by mail to the University of Michigan. Among respondents who: a) were alive in 2006 (since assignment was based on 2004 data), b) were age eligible (older than 50), c) not a nursing home resident, d) completed a non-proxy interview (some interviews were completed by well-informed relatives), and e) received the self-administered questionnaire, the response rate was $90 \%$ (final sample $N=7168$ ). The HRS website provides extensive documentation about the protocol, instrumentation, and complex sampling strategy (http:// hrsonline.isr.umich.edu/). All HRS protocols are reviewed by the Institutional Review Board at the University of Michigan where the study is located and archived (http:// hrsonline.isr.umich.edu/sitedocs/irb/HRS_IRB_Information.pdf). This study used deidentified, publicly available data which can be downloaded from the HRS website.

\section{Measures}

Life Satisfaction Measurement-At baseline (2006), life satisfaction was assessed using the validated Satisfaction with Life Scale, a 5-item measure with responses for each item ranging from 1 (strongly disagree) to 6 (strongly agree) (Diener, Emmons, Larsen, \& Griffin, 1985). All five items were averaged for a final score (average score $=4.37$ (SD $=$ 1.21). The scores were then standardized $(\mu=0, \sigma=1)$ to facilitate interpretation and comparison of effect size across studies.

Indicators of Preventive Health Care Services and Behaviors-Outcome variables were measured in 2008. Each respondent was asked gender-specific questions regarding use of preventive health care services over the last two years (yes/no). In total, HRS asked about six preventive services recommended by either the United States Preventive Services Task Force or the Centers for Disease Control. Respondents were asked: In the last two years, have you had any of the following medical tests or procedures: A flu shot? A blood test for cholesterol? A mammogram or x-ray of the breast to search for cancer? A pap smear? An examination of your prostrate to screen for cancer? HRS asked about one additional preventive measure where a clinic visit was not required: Do you check your breasts for lumps monthly?

To identify visits that were made in the service of primary prevention, the number of respondents in our analyses changed depending on which preventive service we examined. For example, our mammogram analysis used data only from women with no history of cancer. Sensitivity analyses comparing models with and without adjustment for the relevant 
disease (e.g., including and excluding women with a history of cancer in the mammogram analyses) indicated little difference in the estimated effects.

Self-reported health care utilization shows substantial agreement with both administrative claims and medical records (Cleary \& Jette, 1984; Reijneveld \& Stronks, 2001; Ritter et al., 2001). Further, the FIRS preventive measures were evaluated by benchmarking them against other national surveys and shown to have high reliability and validity (Jenkins, Ofstedal, \& Weir, 2008).

\section{Statistical Analyses}

We used logistic regression in all analyses. In the present study, all results can be interpreted as the change in odds of obtaining a preventive service as a function of a one standard deviation increase in life satisfaction score. The overall item non-response rate was $2.29 \%$. Therefore, we used multiple imputation procedures to account for missing data, because this technique provides a more accurate estimate of association than other missing data methods.

Based on prior work that identified relevant covariates, we adjusted for twelve factors (see Table 1). All covariates were measured at baseline via self-report. Due to space limitations, a full explanation of how the covariates were assessed are described in the supplemental methods section. We first examined a non-adjusted model (Model 1). Model 2 included the following sociodemographic factors: age, gender, race/ethnicity, marital status, education level, total wealth. Model 3, a fully adjusted model, added physical and mental health factors (number of previous doctor visits, insurance status, an index of major chronic illnesses, functional status, depression, and anxiety). Table 1 summarizes the findings for these three models. In addition, we created quartiles of life satisfaction based on the score distribution in this sample, in order to consider the possibility of threshold or discontinuous effects.

\section{Results}

The average respondent's age at baseline was 69 years $(\mathrm{SD}=9.84)$. Respondents tended to be female (58\%) and married (65\%). Respondents self-identified as being White (78\%), Black (13\%), Hispanic (7\%) or "Other"(1\%). Most had a high school degree (55\%) or attended some college (26\%). More than $96 \%$ had health insurance. Table S1 describes the distribution of covariates across quartiles of life satisfaction. Analyses that adjusted for sociodemographic factors (Table 1, Model 2) showed that people with higher life satisfaction were not more likely to obtain a preventive flu shot $(\mathrm{OR}=1.11,95 \% \mathrm{CI}=1.05$ to 1.17). However, each standard deviation increase in life satisfaction was associated with a higher likelihood that people would obtain a cholesterol test $(\mathrm{OR}=1.18,95 \% \mathrm{CI}=1.10$ to 1.27). Further, more satisfied women were more likely to obtain a mammogram $/ \mathrm{x}$-ray $(\mathrm{OR}=$ $1.19,95 \% \mathrm{CI}=1.11$ to 1.28$)$ or pap smear $(\mathrm{OR}=1.14,95 \% \mathrm{CI}=1.07$ to 1.22$)$, while more satisfied men were more likely to obtain a prostate exam $(\mathrm{OR}=1.2295 \% \mathrm{CI}=1.11$ to 1.33$)$. Finally, women who reported higher life satisfaction were more likely to report that they regularly checked their breasts for lumps $(\mathrm{OR}=1.07,95 \% \mathrm{CI}=1.00$ to 1.15 ; see Table 1$)$. Results from the unadjusted model (Table1, Model 1) and fully adjusted model (Table1, Model 3) showed largely similar patterns. When evaluating results where life satisfaction 
was split into quartiles, the results hinted at a threshold effect above "low" or "low to moderate" levels of satisfaction with many (but not all) types of preventive services (see Table S2 for detailed information).

\section{Discussion}

Our findings suggest that people with higher life satisfaction are more likely to obtain recommended preventive health care services. Together with other work suggesting that people with higher life satisfaction engage in healthier behaviors (Kim et al., 2014; Li et al., 2009; Strine et al., 2008), this study suggests that one reason satisfied people have better health and make fewer overall doctor visits (Kim et al., 2014) is because they engage in a constellation of health-enhancing behaviors and preventive measures. Further, the association between life satisfaction and preventive health care services persisted even after adjusting for depression and anxiety, which decreases concerns that life satisfaction merely reflects an absence of psychological distress.

Our study had several limitations. For example, health care use was assessed with selfreported data. However, the validity of self-reported health care use has been supported with various samples in past research, whereby self-reported health care use shows high agreement with medical records/administrative claims (Cleary \& Jette, 1984; Reijneveld \& Stronks, 2001; Ritter et al., 2001). There are also several covariates that we did not adjust for that might confound the association between life satisfaction and use of preventive services (e.g., social connectedness and personality variables). Future research should examine these topics.

Health care professionals often have difficulty helping patients change their health behaviors. Although causal conclusions cannot be drawn from observational studies, data from this study suggests that having more life satisfaction may motivate patients to act in healthier ways, and as a result may provide a novel target for intervention. Life satisfaction can and does change (Diener et al., 2006; Lucas \& Donnellan, 2007). A recent meta-analysis found that nine out of twelve randomized controlled trials that targeted life satisfaction were able to successfully modify satisfaction levels (Bolier et al., 2013).

Further, findings with another aspect of well-being, positive affect, suggest that modifying well-being may enhance preventive health behaviors. For example, a series of randomized controlled trials among chronic disease patients compared a group receiving patient education against a group receiving patient education as well as positive affect inductions. The studies found that groups which were supplemented with positive affect inductions showed increased medication adherence and physical activity (Ogedegbe et al., 2012; Peterson et al., 2012). These randomized controlled trials provide a proof-of-concept that raising various facets of well-being can lead to better health behaviors, although more research is needed in older age individuals (e.g., age 50 to 100) to determine which types of interventions are effective (e.g., Proyer, Gander, Wellenzohn \& Ruch, in press). If future studies replicate our findings, a question of pressing concern will be whether interventions that enhance life satisfaction can enhance people's preventive habits. Results from such 
studies may reveal innovative and inherently rewarding ways of improving the health behaviors and health of our aging society.

\section{Supplementary Material}

Refer to Web version on PubMed Central for supplementary material.

\section{Acknowledgments}

We would like to thank the associate editor, editor, and the anonymous reviewers for their valuable comments and suggestions. The HRS (Health and Retirement Study) is sponsored by the National Institute on Aging (grant number NIA U01AG009740) and is conducted by the University of Michigan. Work on this project was supported in part by NIH Grant AG040635.

\section{References}

Boehm JK, Peterson C, Kivimaki M, Kubzansky LD. Heart health when life is satisfying: Evidence from the Whitehall II cohort study. European Heart Journal. 2011; 32:2672-2677.10.1093/eurheartj/ ehr203 [PubMed: 21727096]

Bolier L, Haverman M, Westerhof GJ, Riper H, Smit F, Bohlmeijer E. Positive psychology interventions: A meta-analysis of randomized controlled studies. BMC Public Health. 2013; 13:119.10.1186/1471-2458-13-119 [PubMed: 23390882]

Centers for Disease Control and Prevention. Healthy aging: Helping people to live long and productive lives and enjoy a good quality of life. Atlanta, GA: 2011. Retrieved from http://stacks.cdc.gov/ view/cdc/6114

Cleary PD, Jette AM. The validity of self-reported physician utilization measures. Medical Care. 1984; 22:796-803. [PubMed: 6492908]

Department of Health and Human Services. Healthy people 2020, older adult section. 2010. Retrieved from http://www.healthypeople.gov/2020/topicsobjectives2020/overview. aspx?topicid=31

Diener E, Chan MY. Happy people live longer: Subjective well-being contributes to health and longevity. Applied Psychology: Health and Weil-Being. 2011; 3:1-43.10.1111/ j.1758-0854.2010.01045.x

Diener E, Emmons RA, Larsen RJ, Griffin S. The Satisfaction With Life Scale. Journal of Personality Assessment. 1985; 49:71-75.10.1207/s15327752jpa4901_13 [PubMed: 16367493]

Diener E, Lucas RE, Scollon CN. Beyond the hedonic treadmill: Revising the adaptation theory of well-being. American Psychologist. 2006; 61:305-314.10.1037/0003-066X.61.4.305 [PubMed: 16719675]

Jenkins, KR.; Ofstedal, MB.; Weir, D. Documentation of Health Behaviors and Risk Factors Measured in the Health and Retirement Study (HRS/AHEAD). Ann Arbor, MI: Survey Research Center, University of Michigan; 2008.

Kim ES, Park N, Sun JK, Smith J, Peterson C. Life satisfaction and frequency of doctor visits. Psychosomatic Medicine. 2014; 76:86-93.10.1097/PSY.0000000000000024 [PubMed: 24336427]

Li C, Ford ES, Zhao G, Mokdad AH. Associations of health risk factors and chronic illnesses with life dissatisfaction among U.S. adults: The Behavioral Risk Factor Surveillance System, 2006. Preventive Medicine. 2009; 49:253-259.10.1016/j.ypmed.2009.05.012 [PubMed: 19501613]

Lucas RE, Donnellan MB. How stable is happiness? Using the STARTS model to estimate the stability of life satisfaction. Journal of Research in Personality. 2007; 41:1091-1098. doi:16/j.jrp. 2006.11.005. [PubMed: 18836511]

Ogedegbe GO, Boutin-Foster C, Wells MT, Allegrante JP, Isen AM, Jobe JB, Charlson ME. A randomized controlled trial of positive-affect intervention and medication adherence in hypertensive African Americans. Archives of Internal Medicine. 2012; 172:322-326.10.1001/ archinternmed.2011.1307 [PubMed: 22269592]

Peterson JC, Charlson ME, Hoffman Z, Wells MT, Wong SC, Hollenberg JP, Allegrante JP. A randomized controlled trial of positive-affect induction to promote physical activity after 
percutaneous coronary intervention. Archives of Internal Medicine. 2012; 172:329-336.10.1001/ archinternmed.2011.1311 [PubMed: 22269589]

Proyer RT, Gander F, Wellenzohn S, Ruch W. Positive psychology interventions in people aged 50-79 years: Long-term effects of placebo-controlled online interventions on well-being and depression. Aging \& Mental Health. in press. 10.1080/13607863.2014.899978

Reijneveld SA, Stronks K. The validity of self-reported use of health care across socioeconomic strata: A comparison of survey and registration data. International Journal of Epidemiology. 2001; 30:1407-1414.10.1093/ije/30.6.1407 [PubMed: 11821355]

Ritter PL, Stewart AL, Kaymaz H, Sobel DS, Block DA, Lorig KR. Self-reports of health care utilization compared to provider records. Journal of Clinical Epidemiology. 2001; 54:136-141. doi:16/S0895-4356(00)00261-4. [PubMed: 11166528]

Sin NL, Lyubomirsky S. Enhancing well-being and alleviating depressive symptoms with positive psychology interventions: A practice-friendly meta-analysis. Journal of Clinical Psychology. 2009; 65:467-487.10.1002/jclp.20593 [PubMed: 19301241]

Strine TW, Chapman DP, Balluz LS, Moriarty DG, Mokdad AH. The associations between life satisfaction and health-related quality of life, chronic illness, and health behaviors among U.S. community-dwelling adults. Journal of Community Health. 2008; 33:40-50.10.1007/ s10900-007-9066-4 [PubMed: 18080207]

Xu J, Roberts RE. The power of positive emotions: It's a matter of life or death- subjective wellbeing and longevity over 28 years in a general population. Health Psychology. 2010; 29:9_ 19.10.1037/a0016767 [PubMed: 20063931] 
Table 1

Odds ratios for the association between life satisfaction and preventive health care services

\begin{tabular}{|c|c|c|c|c|}
\hline Preventive Service & Unadjusted Model & Adjusted For Demographics $a$ & Fully Adjusted Model $^{b}$ & Prevalence \\
\hline Flu shot ${ }^{c}$ & $1.11(1.05-1.17)^{* * *}$ & $0.98(0.93-1.03)$ & $1.05(0.99-1.11)$ & $64.75 \%$ \\
\hline Cholesterol test ${ }^{d}$ & $1.18(1.10-1.27)^{* * *}$ & $1.07(1.00-1.16)^{*}$ & $1.12(1.03-1.22)^{* *}$ & $80.93 \%$ \\
\hline Mammogram $^{e}$ & $1.19(1.11-1.28)^{* * *}$ & $1.11(1.03-1.21)^{* *}$ & $1.10(1.01-1.20)^{*}$ & $70.73 \%$ \\
\hline Pap smear ${ }^{e}$ & $1.14(1.07-1.22)^{* * *}$ & $1.14(1.06-1.23)^{* * *}$ & $1.09(1.00-1.18)^{*}$ & $47.73 \%$ \\
\hline Prostate exam $f$ & $1.22(1.11-1.33)^{* * *}$ & $1.11(1.01-1.23)^{*}$ & $1.14(1.03-1.26)^{*}$ & $68.86 \%$ \\
\hline Breast exam ${ }^{e}$ & $1.07(1.00-1.15)^{*}$ & $1.10(1.02-1.18)^{* *}$ & $1.08(1.00-1.17)^{*}$ & $54.83 \%$ \\
\hline \multicolumn{5}{|l|}{ * $<<.05$} \\
\hline \multicolumn{5}{|l|}{ *** $\mathrm{p}<.01$} \\
\hline \multicolumn{5}{|l|}{ **** $\mathrm{p}<.001$} \\
\hline \multicolumn{5}{|c|}{$a_{\text {Demographic factors: age, gender, race/ethnicity, marital status, education level, and total wealth }}$} \\
\hline \multicolumn{5}{|c|}{$\begin{array}{l}b \text { All covariates: age, gender, race/ethnicity, marital status, education level, total wealth, number of previous doctor visits, insurance status, an index } \\
\text { of major chronic illnesses (high blood pressure, diabetes, cancer or malignant tumor of any kind, lung disease, heart attack, coronary heart disease, } \\
\text { angina, congestive heart failure, or other heart problems, emotional, nervous, or psychiatric problems, arthritis or rheumatism, and stroke), } \\
\text { functional status, depression, and anxiety }\end{array}$} \\
\hline \multicolumn{5}{|l|}{$c_{\mathrm{n}=7,168}$} \\
\hline \multicolumn{5}{|c|}{$d_{\text {Excluded people with history of heart disease or stroke }(\mathrm{n}=5,166)}$} \\
\hline \multicolumn{5}{|c|}{$e^{e}$ Included only women with no history of cancer $(\mathrm{n}=3,535)$} \\
\hline
\end{tabular}

